\title{
NONUNIMODALITY OF GRADED GORENSTEIN ARTIN ALGEBRAS
}

\author{
MATS BOIJ AND DAN LAKSOV
}

(Communicated by Eric Friedlander)

\begin{abstract}
We give an explicit expression for the Hilbert function of a large class of graded Gorenstein Artin algebras and give a criterion for this function to be unimodal. As a result we obtain an abundance of graded Gorenstein Artin algebras with nonunimodal Hilbert function.
\end{abstract}

\section{INTRODUCTION}

In this article we shall prove that, given three positive integers $r, s$, and $c$ such that

$$
\frac{f_{r}\left(\left\lfloor\frac{c+1}{2}\right\rfloor\right)+f_{r}\left(\left\lfloor\frac{c+2}{2}\right\rfloor\right)-f_{r}\left(\left\lfloor\frac{c-1}{2}\right\rfloor\right)}{f_{r}\left(\left\lfloor\frac{c-3}{2}\right\rfloor\right)}<s \leq f_{r}(c),
$$

where $f_{r}(d)=\left(\begin{array}{c}r-1+d \\ r-1\end{array}\right)$, then there exists an abundance of graded Gorenstein Artin rings of embedding dimension $r+s$, with socle in degree $c+1$ and having Hilbert function that is not unimodal. In fact, if $s>1$, then there exists a constant $c_{0}$ such that for $c>c_{0}$ all the rings we construct have nonunimodal Hilbert function. We illustrate our result in Example (5.5) with the particular cases of embedding dimension 5 and 13 and show that the simplest counterexamples of R. Stanley [S1] and of D. Bernstein and A. Iarrobino [B-I], to the conjecture that the Hilbert functions of Gorenstein Artin rings are unimodal, are extreme cases (encircled in the figures) in large classes of counterexamples. In fact, they have minimal socle degree and embedding dimension, respectively, in this class.

The basic idea of our construction of graded Gorenstein Artin rings is due to Bernstein and Iarrobino [B-I] and consists in starting with compressed Artin algebras of which we know the Hilbert functions and then to use results of $T$. Gulliksen and I. Reiten, in the same way as was done by Stanley in [S1], to construct graded Gorenstein Artin algebras of which we also know the Hilbert functions.

Our approach differs from that of Bernstein and Iarrobino in that we work directly with algebras and do not use the (Macaulay-Matlis) dual approach via

Received by the editors April 14, 1993.

1991 Mathematics Subject Classification. Primary 13A02; Secondary 13D40, 13 E10.

Key words and phrases. Artin algebra, Gorenstein algebra, Hilbert function, compressed algebra, unimodal. 
differential modules. In an earlier article [F-L] from which we extract our construction of compressed algebras, we demonstrated the advantages of working directly with algebras. The present article further illustrates these advantages. Indeed, our methods lead to a short, self-contained, and direct presentation of the construction of graded Gorenstein Artin algebras with Hilbert function

$$
\min \left\{\left(\begin{array}{c}
r-1+d \\
r-1
\end{array}\right), s\left(\begin{array}{c}
r-1+c-d \\
r-1
\end{array}\right)\right\}+\min \left\{\left(\begin{array}{c}
r+c-d \\
r-1
\end{array}\right), s\left(\begin{array}{c}
r-2+d \\
r-1
\end{array}\right)\right\}
$$

for all positive integers $r, s$, and $c$.

One of the main contributions of the present article is a criterion for functions of the above type to be unimodal when restricted to integers. As a result of the criterion and the construction we obtain the explicit formula presented in the beginning of this introduction.

We would like to thank Ralf Fröberg for useful comments.

\section{ARTIN LEVEL ALGebras}

In this section we shall introduce the basic notation and terminology and prove some useful results on Artin level algebras. We shall also show how to construct graded Gorenstein Artin algebras from graded Artin level algebras.

Setup (2.1). Let $A=A_{0} \oplus A_{1} \oplus \cdots$ be a graded algebra over a field $k=A_{0}$. For any subset $T \subseteq A$ and any integer $d \geq 0$ we write $T_{d}=T \cap A_{d}$. Moreover, for any $k$-subspace $V \subseteq A_{c}$ and every integer $d$ we write

$$
(V: A)_{d}=\left\{a \in A_{d} \mid a b \in V \text { for all } b \in A_{c-d}\right\} .
$$

We then have that $(V: A)_{c}=V$ and $(V: A)_{d}=A_{d}$ for $d>c$. Let $R=$ $k\left[x_{1}, \ldots, x_{r}\right]$ be the polynomial ring in the $r$ variables $x_{1}, x_{2}, \ldots, x_{r}$ over the field $k$.

Definition (2.2). The socle of $A$ is defined by

$$
\operatorname{Soc}(A)=\left\{a \in A \mid a b=0 \text { for all } b \in A_{1} \oplus A_{2} \oplus \cdots\right\} .
$$

Definition (2.3). The graded algebra $A$ is said to be standard if it is generated by $A_{1}$ as a $k$-algebra, and an Artin algebra $A$ is said to be level if it is standard and $\operatorname{Soc}(A)=A_{c}$ for some integer $c$. (For the definition of level algebras of higher Krull-dimensions see [S2].)

Proposition (2.4). Let $A=A_{0} \oplus A_{1} \oplus \cdots$ be a standard graded algebra over a field $k=A_{0}$, and let $I \subseteq A$ be a homogeneous ideal such that $A / I$ is Artin. Then $\operatorname{Soc}(A / I)=(A / I)_{c}$ if and only if $I=\bigoplus_{d=0}^{\infty}(V: A)_{d}$ for some linear subspace $V \subseteq A_{c}$.

In particular, any Artin level algebra over $k$ can be constructed as a quotient of the polynomial ring $R$ by a homogeneous ideal $I=\bigoplus_{d=0}^{\infty}(V: R)_{d}$, where $V$ is a linear subspace of $R_{c}$ for some integer $c$.

Proof. Suppose that $A / I$ is a level Artin algebra with socle in degree $c$. Let $V=I_{c}$. Then we have that $I_{d} \subseteq(V: A)_{d}$ for all integers $d$. Moreover, we have that $I_{c}=(V: A)_{c}$. We shall show, by descending induction on $d$, that $I_{d}=(V: A)_{d}$. Assume, by induction, that $I_{d+1}=(V: A)_{d+1}$ for some $d<c$. Let $a \in(V: A)_{d}$. Then $a b \in V$ for all $b \in A_{c-d}$ and consequently $a b b^{\prime} \in V$ for all $b \in A_{1}$ and $b^{\prime} \in A_{c-d-1}$. Hence we have that $a b \in(V: A)_{d+1}=I_{d+1}$ 
for all $b \in A_{1}$. Since $A$ is a standard algebra, it follows that the class of $a$ is in $\operatorname{Soc}(A / I)$, and since $\operatorname{Soc}(A / I)=(A / I)_{c}$, we must have that $a \in I_{d}$. We have proved that $I_{d} \supseteq(V: A)_{d}$ and hence that $I=\bigoplus_{d=0}^{\infty}(V: A)_{d}$.

Conversely, it is easy to show that if $I=\bigoplus_{d=0}^{\infty}(V: A)_{d}$ for some linear subspace $V \subseteq A_{c}$ we have, that $\operatorname{Soc}(A / I)=(A / I)_{c}$.

Definition (2.5). Given a graded Artin algebra $A=A_{0} \oplus A_{1} \oplus \cdots \oplus A_{c}$ over a field $k=A_{0}$. Let $E=\operatorname{Hom}_{k}(A, k)$ be the space of $k$-linear maps from $A$ to $k$. Then $E=E_{1} \oplus E_{2} \oplus \cdots \oplus E_{c+1}$ where $E_{d}=\operatorname{Hom}_{k}\left(A_{c+1-d}, k\right)$. We give $E$ an $A$-module stucture by $(x \phi)(y)=\phi(x y)$, for $x, y \in A$ and $\phi \in E$. Then $E$ becomes a graded $A$-module. We introduce a commutative ring structure on the cartesian product $S=A \times E$ by $(x, \phi)+(y, \psi)=(x+y, \phi+\psi)$ and $(x, \phi)(y, \psi)=(x y, x \psi+y \phi)$. The gradings of $A$ and $E$ induce a grading of $S=A \times E$ such that $S_{d}=A_{d} \times E_{d}=A_{d} \times \operatorname{Hom}_{k}\left(A_{c+1-d}, k\right)$. We have that $S_{d}=0$ for $d>c+1$ and $S_{c+1}=\operatorname{Hom}_{k}(k, k) \cong k$.

Lemma (2.6). Assume that $A$ is Artin level with socle in degree $c$. Then, for any nonnegative integers $i, j$ with $i+j \leq c$, the natural map $\phi_{i j}: A_{i} \rightarrow$ $\operatorname{Hom}_{k}\left(A_{j}, A_{i+j}\right)$ is injective.

Proof. Assume that there is a nonnegative integer $j$ such that $i+j \leq c$ and such that $\phi_{i j}$ is not injective, and denote by $j^{\prime}$ the smallest such $j$. Then $j^{\prime}>0$. Choose an element $a$ in the kernel of $\phi_{i j^{\prime}}$. Then we have that $a b=0$ for all $b \in A_{j^{\prime}}$ and hence $a b^{\prime} c=0$ for all $b^{\prime} \in A_{j^{\prime}-1}$ and all $c \in A_{1}$. Since $A$ is level and $i+j^{\prime} \leq c$, this implies that $a b^{\prime}=0$ for all $b^{\prime} \in A_{j^{\prime}-1}$, contradicting the minimality of $j^{\prime}$. Hence $\operatorname{ker} \phi_{i j}=0$ for all nonnegative integers $i, j$ such that $i+j \leq c$.

Lemma (2.7). Assume that $A$ is Artin level with socle in degree $c$. Then the graded $k$-algebra $S=A \times E$ is Artin and standard.

Proof. Since $S$ is a finite-dimensional $k$-vector space, it has Krull-dimension zero. Since $A$ is standard, it is sufficient to show that elements in $\operatorname{Hom}_{k}(A, k)$ are in the sub- $A$-algebra of $S$ generated by $S_{1}$. That is, it suffices to show that, for all $1 \leq d \leq c+1$, the map $A_{d-1} \otimes E_{1} \rightarrow E_{d}$ given by the module structure on $E$ is surjective. The latter map is surjective if and only if the dual map $E_{d}^{*} \rightarrow A_{d-1}^{*} \otimes E_{1}^{*}$ is injective. But the latter is the natural map $A_{c+1-d} \rightarrow \operatorname{Hom}_{k}\left(A_{d-1}, A_{c}\right)$ which is injective by Lemma (2.6).

Proposition (2.8). Assume that $A$ is a graded Artin level algebra with socle in degree $c$. Then the graded $k$-algebra $S=A \times E$ is level with a one-dimensional socle in degree $c+1$.

Proof. By Lemma (2.7), $S$ is standard and Artin. Suppose that $(x, \phi) \in$ $\operatorname{Soc}(S)$. Then $x \psi=0$ for all $\psi \in E$ which implies that $x=0$. Furthermore, we have that $y \phi=0$ for all $y \in A_{1} \oplus A_{2} \oplus \cdots$ which implies that $\phi(y)=0$ for all $y \in A_{1} \oplus A_{2} \oplus \cdots$, and consequently we have that $\phi \in \operatorname{Hom}_{k}\left(A_{0}, k\right)=E_{c+1}$. Thus $\operatorname{Soc}(S) \subseteq S_{c+1}$ and $S$ is level.

Remark (2.9). Recall that an Artin algebra is Gorenstein if it is level and the socle is one dimensional. Hence Proposition (2.8) states that $S=A \times E$ is Gorenstein if $A$ is level. For local rings a corresponding result was proved by Gulliksen [G, Lemma, p. 231] and Reiten [R, Corollary 6]. By general principles [H-R] it carries over to graded rings (see [S1, Example 4.3]). 


\section{COMPRESSED ALGEBRAS}

In this section we give a self-contained construction of level Artin compressed algebras. The material is extracted from [F-L].

Setup (3.1). Let $R=k\left[x_{1}, \ldots, x_{r}\right]$ be the polynomial ring in the $r$ independent variables $x_{1}, x_{2}, \ldots, x_{r}$ over an infinite field $k$, and let $M$ be the monomial $k$-basis for $R$.

Given a subspace $V$ of $R_{c}$ and a subspace $W$ of $R_{d}, W \cap(V: R)_{d}$ is the kernel of the map

$$
W \rightarrow \bigoplus_{g \in M_{c-d}}\left(R_{c} / V\right)
$$

sending $f \in W$ to $\bigoplus_{g \in M_{c-d}} g f$. Therefore, we have that

$$
\operatorname{codim}_{k}\left(W \cap(V: R)_{d}, W\right) \leq \min \left\{\operatorname{dim}_{k} W, \operatorname{dim}_{k} R_{c-d} \operatorname{codim}_{k}\left(V, R_{c}\right)\right\} .
$$

The main result of this section is that, if $V$ is a subspace of $R_{c}$ in general position, then the above inequality is in fact an equality. More precisely, for all integers $t$ such that $1 \leq t \leq \operatorname{dim}_{k} R_{c}$, equality holds for all subspaces $V$ corresponding to points in an open nonempty set in the Grassmannian of $t$ dimensional subspaces of $R_{c}$.

Lemma (3.2). Given a subspace $W$ in $R_{d}$, the following assertions hold:

(i) For $n$ points $a_{1}, a_{2}, \ldots, a_{n}$ in general position in the r-dimensional affine space $\mathbf{A}_{k}^{r}$ over $k$ we have that

$$
\operatorname{codim}_{k}\left(\left\{f \in W \mid f\left(a_{i}\right)=0,1 \leq i \leq n\right\}, W\right)=\min \left\{\operatorname{dim}_{k} W, n\right\} .
$$

(ii) Let $N=\operatorname{dim}_{k} R_{c-d}$, and choose points $a_{1}, a_{2}, \ldots, a_{N}$ in $\mathbf{A}_{k}^{r}$ such that

$$
\operatorname{codim}_{k}\left(\left\{g \in R_{c-d} \mid g\left(a_{i}\right)=0,1 \leq i \leq N\right\}, R_{c-d}\right)=N .
$$

Define a hyperplane $H$ in $R_{c}$ by $H=\left\{h \in R_{c} \mid \sum_{i=1}^{N} h\left(a_{i}\right)=0\right\}$. Then

$$
W \cap(H: R)_{d}=\left\{f \in W \mid f\left(a_{i}\right)=0,1 \leq i \leq N\right\} .
$$

Proof. The first statement of the lemma is proved by induction on $n$. Assume that we have found points $a_{1}, a_{2}, \ldots, a_{j}$ such that

$$
\operatorname{codim}_{k}\left(\left\{f \in W \mid f\left(a_{i}\right)=0,1 \leq i \leq j\right\}, W\right)=j
$$

for some $j<\operatorname{dim}_{k} W$. Then $\operatorname{dim}_{k}\left\{f \in W \mid f\left(a_{i}\right)=0,1 \leq i \leq j\right\}=\operatorname{dim}_{k} W-$ $j>0$. Since the field $k$ is infinite, we can find a point $a_{j+1}$ in $\mathbf{A}_{k}^{r}$ such that $g\left(a_{j+1}\right) \neq 0$ for some polynomial $g \in\left\{f \in W \mid f\left(a_{i}\right)=0,1 \leq i \leq j\right\}$. Then we have that

$$
\operatorname{codim}_{k}\left(\left\{f \in W \mid f\left(a_{i}\right)=0,1 \leq i \leq j+1\right\}, W\right)=j+1 .
$$

Hence the first assertion of the lemma holds.

To prove the second statement of the lemma we observe that the condition (3.2.1) means that the system of equations $\sum_{g \in M_{c-d}} y_{g} g\left(a_{i}\right)=0$ for $i=1,2, \ldots, N$ in the $N$ variables $y_{g}$ has no nontrivial solution. By the definition of the hyperplane $H$ we have that

$$
(H: R)_{d}=\left\{f \in R_{d} \mid \sum_{i=1}^{N} f\left(a_{i}\right) g\left(a_{i}\right)=0, \text { for all } g \in M_{c-d}\right\} .
$$


Hence we have the equality $(H: R)_{d}=\left\{f \in R_{d} \mid f\left(a_{i}\right)=0,1 \leq i \leq N\right\}$ of which the second assertion of the lemma is an immediate consequence.

Proposition (3.3). Given a subspace $W$ in $R_{d}$, if $V$ is a subspace of $R_{c}$ in general position, then

$$
\operatorname{codim}_{k}\left(W \cap(V: R)_{d}, W\right)=\min \left\{\operatorname{dim}_{k} W, \operatorname{dim}_{k} R_{c-d} \operatorname{codim}_{k}\left(V, R_{c}\right)\right\}
$$

and the space $(V: R)_{d}$ intersects $W$ properly.

Proof. From general principles it follows that it is sufficient to prove the proposition for one subspace $V$ of each codimension in $R_{c}$. Furthermore, it is sufficient to prove the proposition for hyperplanes. In fact, we can then choose hyperplanes $H_{1}, H_{2}, \ldots, H_{t}$ by induction on $t$ such that

$$
\operatorname{codim}_{k}\left(W \cap\left(H_{i+1}: R\right)_{d}, W\right)=\min \left\{\operatorname{dim}_{k} W, \operatorname{dim}_{k} R_{c-d}\right\}
$$

and such that $H_{i+1}$ intersects $W \cap\left(\bigcap_{j=1}^{i} H_{j}: R\right)_{d}=W \cap \bigcap_{j=1}^{i}\left(H_{j}: R\right)_{d}$ properly. Then the subspace $V=\bigcap_{j=1}^{t} H_{j}$ satisfies the conditions of the proposition.

To prove the proposition for hyperplanes let $N=\operatorname{dim}_{k} R_{c-d}$ and choose points $a_{1}, a_{2}, \ldots, a_{N}$ in $\mathbf{A}_{k}^{r}$ such that condition (3.2.1) of Lemma (3.2) is satisfied and such that

$$
\operatorname{codim}_{k}\left(\left\{f \in R_{d} \mid f\left(a_{i}\right)=0,1 \leq i \leq N\right\}, R_{d}\right)=\min \left\{\operatorname{dim}_{k} R_{d}, N\right\}
$$

and

$$
\operatorname{codim}_{k}\left(\left\{f \in W \mid f\left(a_{i}\right)=0,1 \leq i \leq N\right\}, R_{d}\right)=\min \left\{\operatorname{dim}_{k} W, N\right\} .
$$

Now define the hyperplane $H=\left\{h \in R_{c} \mid \sum_{i=1}^{N} h\left(a_{i}\right)=0\right\}$ as in Lemma (3.2). By the same lemma we get equalities

$$
(H: R)_{d}=\left\{f \in R_{d} \mid f\left(a_{i}\right)=0,1 \leq i \leq N\right\}
$$

and

$$
W \cap(H: R)_{d}=\left\{f \in W \mid f\left(a_{i}\right)=0,1 \leq i \leq N\right\} .
$$

It follows from (3.3.3) that (3.3.2) is the equality of the proposition for the subspace $V=H$. Moreover, it follows from (3.3.3) and (3.3.1) that the intersection between $W$ and $(H: R)_{d}$ is proper. Hence we have proved the proposition. sult.

Combining Proposition (3.3) and Proposition (2.4) we get the following re-

Theorem (3.4). Let $V$ be a subspace of $R_{c}$ in general position, and let $I=$ $\bigoplus_{d=1}^{\infty}(V: R)_{d}$. Then $R / I$ is an Artin standard level algebra with socle $R_{c} / V$ and

$$
\operatorname{dim}_{k}(R / I)_{d}=\min \left\{\operatorname{dim}_{k} R_{d}, \operatorname{dim}_{k} R_{c-d} \operatorname{dim}_{k} R_{c} / V\right\} .
$$

\section{A UNIMODALITY CRITERION}

We shall in this section give a criterion for a particular symmetric function to be unimodal. 
Setup (4.1). Let $f, g:[0, a] \rightarrow \mathbf{R}$ be two monotone strictly convex functions. We assume that $f$ is increasing and that $g$ is decreasing and define $h:[0, a] \rightarrow$ $\mathbf{R}$ by

$$
h(t)=\min \{f(t), g(t)\}+\min \{f(a-t), g(a-t)\} .
$$

Lemma (4.2). The function $h$ is symmetric with respect to $t=a / 2$, and there is a real number $t_{0} \in[0, a / 2]$ such that $h$ is strictly increasing on $\left[0, t_{0}\right]$ and strictly decreasing on $\left[t_{0}, a / 2\right]$.

In particular, $h$ has no more than two local maxima and the only possible local minima are $t=0, t=a / 2$, and $t=a$.

Proof. That $h$ is symmetric with respect to $a / 2$ follows immediately from its definition. There is a number $b \in[0, a]$ such that

$$
\min \{f(t), g(t)\}= \begin{cases}f(t) & \text { if } 0 \leq t<b \\ g(t) & \text { if } b<t \leq a\end{cases}
$$

Now let $t_{0}=\min \left\{b, t_{0}-b\right\}$. This number has the property that $h$ is strictly increasing on $\left[0, t_{0}\right]$ and strictly decreasing on $\left[t_{0}, a / 2\right]$ since on the interval $\left[0, t_{0}\right] h$ is a sum of two strictly increasing functions and on the interval $\left[t_{0}, a-t_{0}\right] h$ is strictly convex and symmetric around $t=a / 2$.
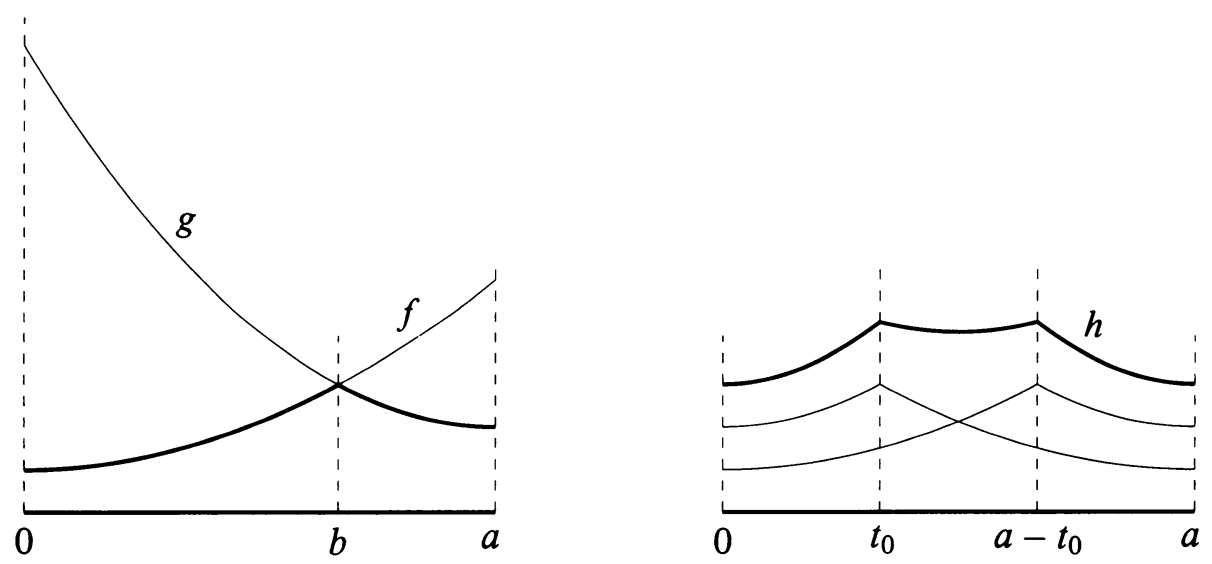

FIGURE (4.3)

Definition (4.4). Let $a$ be an integer, and let $l:[0, a] \rightarrow \mathbf{R}$ be a function satisfying $l(t)=l(a-t)$ for all $t \in[0, a]$. Then we say that $l$ is unimodal on integers if its restriction to the integers $0,1, \ldots, a$ is unimodal, that is, if $l(0) \leq l(1) \leq \cdots \leq l(\lfloor a / 2\rfloor)$.

Lemma (4.5). Assume that $a \geq 2$. Then the function $h$ is unimodal on integers if and only if $h(\lfloor a / 2\rfloor-1) \leq \bar{h}(\lfloor a / 2\rfloor)$.

Proof. Suppose that $h(\lfloor a / 2\rfloor-1) \leq h(\lfloor a / 2\rfloor)$. Since $h$ is symmetric, it can not be strictly convex on the interval from $\lfloor a / 2\rfloor-1$ to $\lceil a / 2\rceil+1$. Hence, by Lemma (4.2), $h$ is increasing from $t=0$ to $t=\lfloor a / 2\rfloor-1$ and, hence, from $t=0$ to $t=\lfloor a / 2\rfloor$, which proves that $h$ is unimodal on integers.

Conversely, if $h(\lfloor a / 2\rfloor-1)>h(\lfloor a / 2\rfloor)$, then, by definition, $h$ is not unimodal on integers. 
Let $r, c$, and $s$ be positive integers. Define the function $f_{r}: \mathbf{R} \rightarrow \mathbf{R}$ by

$$
f_{r}(t)=(1+t)\left(1+\frac{t}{2}\right) \cdots\left(1+\frac{t}{r-1}\right) .
$$

Then $f_{r}$ is a positive strictly convex increasing function on $[-1, \infty)$ when $r>$ 2. We apply the above results to the case when $f(t)=f_{r}(t)$ on $[0, c+1]$ and $g(t)=s f_{r}(c-t)$ on $[0, c+1]$ and with $a=c+1$. If $r>2$, these functions are strictly convex increasing and decreasing, respectively, on the interval $[0, c+1]$.

Define $h_{r c s}:[0, c+1] \rightarrow \mathbf{R}$ by

$$
h_{r c s}(t)=\min \left\{f_{r}(t), s f_{r}(c-t)\right\}+\min \left\{f_{r}(c+1-t), s f_{r}(t-1)\right\} .
$$

Proposition (4.6). Given integers $r>2$ and $c \geq 1$, the function $h_{r c s}$ is unimodal on integers if and only if

$$
s \leq \frac{f_{r}\left(\left\lfloor\frac{c+1}{2}\right\rfloor\right)+f_{r}\left(\left\lfloor\frac{c+2}{2}\right\rfloor\right)-f_{r}\left(\left\lfloor\frac{c-1}{2}\right\rfloor\right)}{f_{r}\left(\left\lfloor\frac{c-3}{2}\right\rfloor\right)} .
$$

When $h_{\text {rcs }}$ is not unimodal on integers, it has exactly two maxima.

Proof. We have assumed that $r>2$. Hence $f_{r}$ is strictly convex on the interval $[-1, c+1]$. By Lemma (4.5), we have to check when $h_{r c s}(\lfloor(c-1) / 2\rfloor) \leq$ $h_{r c s}(\lfloor(c+1) / 2\rfloor)$. We distinguish two cases; $c$ odd and $c$ even.

Case 1. $c$ odd. Let $a_{i}=f_{r}(i+(c-3) / 2)$, for $i=0,1,2,3$. Since $f_{r}((c-3) / 2) \geq f_{r}(-1)=0$ and since $f_{r}$ is strictly convex, we have the following inequalities:

$$
a_{0} \geq 0 \text { and } 0<a_{1}-a_{0}<a_{2}-a_{1}<a_{3}-a_{2}
$$

which we shall use extensively. Furthermore, we have that

$$
h_{r c s}\left(\frac{c-1}{2}\right)=\min \left\{a_{1}, s a_{2}\right\}+\min \left\{a_{3}, s a_{0}\right\}=a_{1}+\min \left\{a_{3}, s a_{0}\right\},
$$

since $a_{1}<a_{2} \leq s a_{2}$, and

$$
h_{r c s}\left(\frac{c+1}{2}\right)=2 \min \left\{a_{2}, s a_{1}\right\} .
$$

If $s a_{1} \leq a_{2}$, we have that $h_{r c s}((c+1) / 2)=2 s a_{1}$, so that

$$
h_{r c s}\left(\frac{c-1}{2}\right) \leq a_{1}+s a_{0}<a_{1}+s a_{1} \leq 2 s a_{1}=h_{r c s}\left(\frac{c+1}{2}\right),
$$

and if $s a_{0} \geq a_{3}$, we have that

$$
h_{r c s}\left(\frac{c-1}{2}\right)=a_{1}+a_{3}>2 a_{2} \geq h_{r c s}\left(\frac{c+1}{2}\right) .
$$

Finally, if we simultaneously have that $a_{2}<s a_{1}$ and $s a_{0}<a_{3}$, we have $h_{r c s}((c-1) / 2)=a_{1}+s a_{0}$ and $h_{r c s}((c+1) / 2)=2 a_{2}$. Hence we have that $h_{r c s}((c-1) / 2) \leq h_{r c s}((c+1) / 2)$ if and only if $a_{1}+s a_{0} \leq 2 a_{2}$. We notice that the above three conditions on $s$ are mutually compatible because, as is easily checked,

$$
\frac{a_{2}}{a_{1}}<\frac{2 a_{2}-a_{1}}{a_{0}}<\frac{a_{3}}{a_{0}} \text {. }
$$


Consequently we have proved that $h_{r c s}((c-1) / 2) \leq h_{r c s}((c+1) / 2)$ if and only if $s \leq\left(2 a_{2}-a_{1}\right) / a_{0}$ which is equivalent to

$$
s \leq \frac{2 f_{r}\left(\frac{c+1}{2}\right)-f_{r}\left(\frac{c-1}{2}\right)}{f_{r}\left(\frac{c-3}{2}\right)} .
$$

Case 2. $c$ even. Let $a_{i}=f_{r}(i+c / 2-2)$, for $i=0,1,2,3,4$. Since $f_{r}(c / 2-2) \geq f_{r}(-1)=0$ and since $f_{r}$ is strictly convex, we have the following inequalities:

$$
a_{0} \geq 0 \text { and } 0<a_{1}-a_{0}<a_{2}-a_{1}<a_{3}-a_{2}<a_{4}-a_{3} .
$$

Furthermore, we have that

$$
h_{r c s}\left(\frac{c}{2}\right)=\min \left\{a_{2}, s a_{2}\right\}+\min \left\{a_{3}, s a_{1}\right\}=a_{2}+\min \left\{a_{3}, s a_{1}\right\}
$$

and

$$
h_{r c s}\left(\frac{c}{2}-1\right)=\min \left\{a_{1}, s a_{3}\right\}+\min \left\{a_{4}, s a_{0}\right\}=a_{1}+\min \left\{a_{4}, s a_{0}\right\} .
$$

If $s a_{1} \leq a_{3}$, we have that

$$
h_{r c s}\left(\frac{c}{2}-1\right) \leq a_{1}+s a_{0}<a_{2}+s a_{1}=h_{r c s}\left(\frac{c}{2}\right),
$$

and if $s a_{0} \geq a_{4}$, we have that

$$
h_{r c s}\left(\frac{c}{2}\right) \leq a_{2}+a_{3}<a_{1}+a_{4}=h_{r c s}\left(\frac{c}{2}-1\right) .
$$

Finally, if we simultaneously have that $a_{3}<s a_{1}$ and $s a_{0}<a_{4}$, we have $h_{r c s}(c / 2-1)=a_{1}+s a_{0}$ and $h_{r c s}(c / 2)=a_{2}+a_{3}$. Hence we have that $h_{r c s}(c / 2-1) \leq h_{r c s}(c / 2)$ if and only if $a_{1}+s a_{0} \leq a_{2}+a_{3}$. We notice that the above three conditions on $s$ are mutually compatible because, as is easily checked,

$$
\frac{a_{3}}{a_{1}}<\frac{a_{2}+a_{3}-a_{1}}{a_{0}}<\frac{a_{4}}{a_{0}} .
$$

Consequently we have proved that $h_{r c s}(\lfloor(c-1) / 2\rfloor) \leq h_{r c s}(\lfloor(c+1) / 2\rfloor)$ if and only if $s \leq\left(a_{2}+a_{3}-a_{1}\right) / a_{0}$ which is equivalent to

$$
s \leq \frac{f_{r}\left(\frac{c}{2}\right)+f_{r}\left(\frac{c}{2}+1\right)-f_{r}\left(\frac{c}{2}-1\right)}{f_{r}\left(\frac{c}{2}-2\right)} .
$$

We conclude the proof by observing that (4.6.1) and (4.6.2) together confirm the assertion of the proposition.

\section{Hilbert fUNCTIONS OF GORENSTEIN ARTIN ALGEBRAS}

In this section we sum up the results of the previous sections and give a precise analysis of the unimodality of the Hilbert functions of the graded Gorenstein Artin algebras that we constructed in $\S \S 2$ and 3.

Setup (5.1). Let $R=k\left[x_{1}, x_{2}, \ldots, x_{r}\right]$ be the polynomial ring in the $r$ variables $x_{1}, \ldots, x_{r}$ over an infinite field $k$. 
Proposition (5.2). For any three positive integers $r, s$, and $c$ there are graded Gorenstein Artin algebras $S$ with socle in degree $c+1$ such that

$$
\operatorname{dim}_{k} S_{d}=\min \left\{\operatorname{dim}_{k} R_{d}, s \operatorname{dim}_{k} R_{c-d}\right\}+\min \left\{\operatorname{dim}_{k} R_{c+1-d}, s \operatorname{dim}_{k} R_{d-1}\right\} .
$$

The embedding dimension of $S$ is $r+s$ if $s \leq\left(\begin{array}{c}r-1+c \\ r-1\end{array}\right)$.

Proof. It follows from Theorem (3.4) that we can find level algebras $A$ such that $\operatorname{dim}_{k} A_{d}=\min \left\{\operatorname{dim}_{k} R_{d}, s \operatorname{dim}_{k} R_{c-d}\right\}$. Now, by Proposition (2.8) we have that $S=A \times E$ is the desired Gorenstein algebra.

We now apply Proposition (4.6) to the Hilbert functions of the Gorenstein Artin algebras of Proposition (5.2).

Proposition (5.3). If $r, s$, and $c$ are positive integers and $r>2$, then the Hilbert functions of the graded Gorenstein Artin algebras of Proposition (5.2) are unimodal if and only if

$$
s \leq \frac{f_{r}\left(\left\lfloor\frac{c+1}{2}\right\rfloor\right)+f_{r}\left(\left\lfloor\frac{c+2}{2}\right\rfloor\right)-f_{r}\left(\left\lfloor\frac{c-1}{2}\right\rfloor\right)}{f_{r}\left(\left\lfloor\frac{c-3}{2}\right\rfloor\right)}
$$

where $f_{r}(d)=\operatorname{dim}_{k} R_{d}=\left(\begin{array}{c}r-1+d \\ r-1\end{array}\right)$. When the Hilbert function is not unimodal, it has exactly two maxima.

Remark (5.4). (i) We note that the right-hand side of (5.3.1) approaches 1 as $c$ increases. Hence for all $s>1$ and $r>2$ there is a constant $c_{0}$ such that the Hilbert function in Proposition (5.2) is nonunimodal if $c>c_{0}$.

(ii) Lemma 3 of [B-I], when appropriately corrected, implies the second part of Proposition (5.3).

Example (5.5). It was previously conjectured that the Hilbert function of any graded Gorenstein Artin algebra is unimodal. The first counterexample to this conjecture was given by Stanley [S1, Example 4.3]. Stanley's example is obtained from Proposition (5.2) when $r=3, s=10$, and $c=3$. In this case the embedding dimension is 13. In [B-I, Example 3] Bernstein gives an example of an Gorenstein Artin algebra, of embedding dimension five, which has a nonunimodal Hilbert function. This example is obtained from Proposition (5.2) when $r=3, s=2$, and $c=15$. In Figure (5.6) we have illustrated Proposition (5.3) for embedding dimensions 13 and 5. The thick points correspond to nonunimodal Hilbert functions, and the examples of Stanley and Bernstein are indicated with circles.

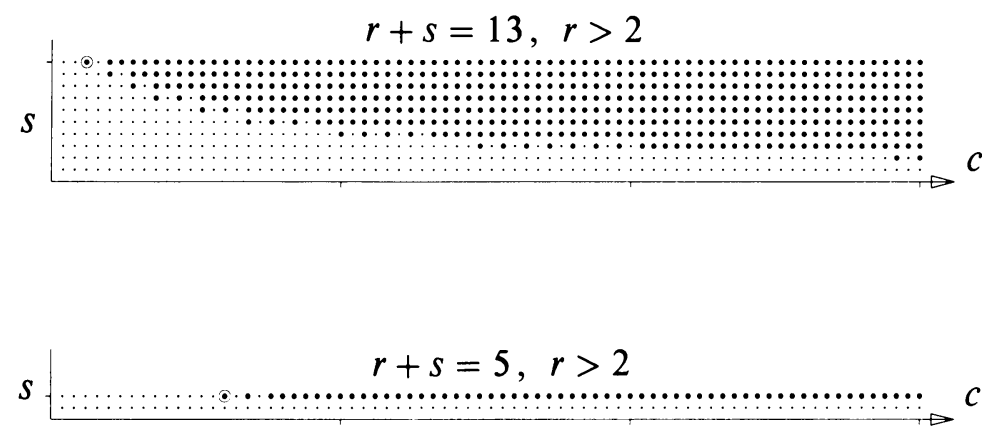

FIGURE (5.6) 


\section{REFERENCES}

[B-I] D. Bernstein and A. Iarrobino, A nonunimodal graded Gorenstein Artin algebra in codimension five, Comm. Algebra 20 (1992), 2323-2336.

[F-L] R. Fröberg and D. Laksov, Compressed algebras, Complete Intersections Acireale 1983, Lecture Notes in Math., vol. 1092, Springer-Verlag, Berlin, 1984, pp. 121-151.

[G] T. H. Gulliksen, Massey operations and the Poincaré series of certain local rings, J. Algebra 22 (1972), 223-232.

[H-R] M. Hochster and L. J. Ratliff, Jr., Five theorems on Macaulay rings, Pacific J. Math. 44 (1973), 147-172.

[I] A. Iarrobino, Compressed algebras: Artin algebras having given socle degrees and maximal length, Trans Amer. Math. Soc. 285 (1984), 337-378.

[R] I. Reiten, The converse to a theorem of Sharp on Gorenstein modules, Proc. Amer. Math. Soc. 32 (1972), 417-420.

[S1] R. Stanley, Hilbert functions of graded algebras, Adv. Math. 28 (1978), 57-83.

[S2] _ Cohen-Macaulay complexes, Higher Combinatorics (M. Aigner, ed.), Reidel, Dordrecht and Boston, 1977, pp. 51-62.

Department of Mathematics, Royal Institute of Technology, S-100 44 Stockholm, SWEDEN

E-mail address: boij@math.kth.se

E-mail address: laksovQmath.kth.se 